\title{
Captura histórica de peces en el Sistema Lagunar Huave, Oaxaca, México
}

\author{
Historical fish catch in the Huave Lagoon System, Oaxaca, \\ Mexico
}

\author{
Pedro Cervantes-Hernández ${ }^{1 *}$, María del Carmen Alejo-Plata ${ }^{1}$, Antonio López-Serrano ${ }^{1}$ \\ y María Isabel Gallardo-Berumen ${ }^{2}$
}

\begin{abstract}
RESUMEN
En el Sistema Lagunar Huave (SLH), la zona Huave está constituida por cuatro cuerpos lagunares: Laguna Mar Tileme y áreas adyacentes, Laguna Inferior y las lagunas Oriental-Occidental. Con los registros mensuales de la captura de peces (kg) y el esfuerzo de pesca (días de pesca), se estimó de 1999 al 2007 un índice denominado "presión por pesca", para evaluar en aquellas, la disponibilidad potencial (DP) y el nivel de explotación (NE) para este recurso. En la Laguna Mar Tileme y áreas adyacentes, la "presión por pesca" resultó "baja". En la Laguna Inferior, la "presión por pesca" fue "alta". En las lagunas Oriental-Occidental, la "presión por pesca" resultó con magnitudes intermedias. Los resultados se compararon con la hidrología del SLH documentada de mayo a agosto / octubre de 2007, evidenciando que donde aquella fue persistentemente extrema (44 - 54.7 UPS, 29.2 - 30 ${ }^{\circ} \mathrm{C}, 5.0$ - $6.7 \mathrm{mg} / \mathrm{l}$ Oxígeno Disuelto (OD)), la DP y el NE fueron menores (en la Laguna Mar Tileme y áreas adyacentes) y donde la hidrología fue persistentemente media (22.5 - 30.0 UPS, $27.0-28.1^{\circ} \mathrm{C}, 7.6-8.0 \mathrm{mg} / \mathrm{lOD}$ ), la DP y el NE fueron mayores (en la Laguna Inferior). Magnitudes intermedias para la DP y el NE se registraron donde la hidrología fue óptima (11.1 - 22.5 UPS, 27.0 - 28.6 ${ }^{\circ} \mathrm{C}, 6.7$ - $7.6 \mathrm{mg} / \mathrm{OOD}$ ) (en las lagunas Oriental-Occidental).
\end{abstract}

Palabras claves: Huave, peces, lagunas costeras, pesquería, Golfo de Tehuantepec.

\begin{abstract}
In the Huave Lagoon System (HLS), the Huave area is comprised of four lagoon bodies: the Mar Tileme Lagoon and adjacent areas, the Inferior Lagoon and the Oriental - Occidental Lagoons. Based on monthly records of fish catches $(\mathrm{Kg})$ and fishing effort (days of fishing), the "fishing pressure" index was estimated from 1999 to 2007 in order to evaluate the potential availability (PA) and exploitation level (EL) for this resource. In the Mar Tileme Lagoon and adjacent areas, the "fishing pressure" was low. In the Inferior Lagoon, the "fishing pressure" was high, while in the Oriental - Occidental Lagoons it showed intermediate magnitudes. Results were compared with HLS documented hydrology from May to August / October 2007, evidencing that where hydrology was persistently extreme (44 - 54.7 PSU, 29.2 - 30 ${ }^{\circ} \mathrm{C}, 5.0$ - $6.7 \mathrm{mg} / 1$ Dissolved Oxygen (DO)), PA and EL were lower (in the Mar Tileme Lagoon and adjacent areas), and where hydrology was persistently intermediate (22.5 - 30.0 PSU, $27.0-28.1^{\circ} \mathrm{C}, 7.6-8.0 \mathrm{mg} / \mathrm{DO}$ ), PA and EL were higher (in the Inferior Lagoon). Intermediate magnitudes for PA and EL were recorded where hydrology was persistently optimal (11.1 - 22.5 PSU, 27.0 - 28.6 ${ }^{\circ} \mathrm{C}, 6.7$ - $7.6 \mathrm{mg} / \mathrm{l} \mathrm{DO}$ ) (in the Oriental - Occidental Lagoons).
\end{abstract}

Keywords: Huave, fish, coastal lagoons, fishery, Gulf of Tehuantepec.

Universidad del Mar, campus Puerto Ángel, Instituto de Recursos. Carretera a Zipolite, Ciudad Universitaria s/n, Distrito de San Pedro Pochutla A. P. 47, C.P. 70902 Puerto Ángel, Oaxaca, México.pch@angel.umar.mx*

2 Egresada de Maestría en Ecología Marina, Universidad del Mar, campus Puerto Ángel, Oaxaca, México.

Recibido: 30 de marzo de 2014

Corregido: 20 de junio de 2014

Aceptado: 18 de julio de 2014

DOI: http://dx.doi.org/10.15359/revmar.6.9

Rev. Mar. Cost. ISSN 1659-455X. Vol. 6: 135-148, Diciembre 2014. 


\section{INTRODUCCIÓN}

En el Sistema Lagunar Huave (SLH) (Fig. 1), desde el año 2001, se reportó una tendencia descendente en el nivel de captura $\left(C_{t}\right)$ para los recursos camarón (Cervantes-Hernández et al. 2006; Cervantes-Hernández et al. 2012) y peces (Fig. 2); que según diferentes autores, aquella ha sido ocasionada principalmente por problemas de índole sociocultural (Bozada-Robles, 2002; Espinoza-Tenorio et al. 2010; Espinoza-Tenorio et al. 2013).
En las zonas Huave (Mar Tileme y áreas adyacentes, Laguna Inferior, Laguna Oriental, Laguna Occidental) y Zapoteca (Laguna Superior) (Fig. 1), la pesca artesanal de peces y camarón se rige por el sistema de "Usos y Costumbres", con un alto sentido de la pertenencia étnica (Millán, 2003) y resistencia al impacto por la influencia entre comunidades e intromisiones externas (Espinoza-Tenorio et al. 2011). La problemática sociocultural vivida en el SLH ha retrasado por décadas

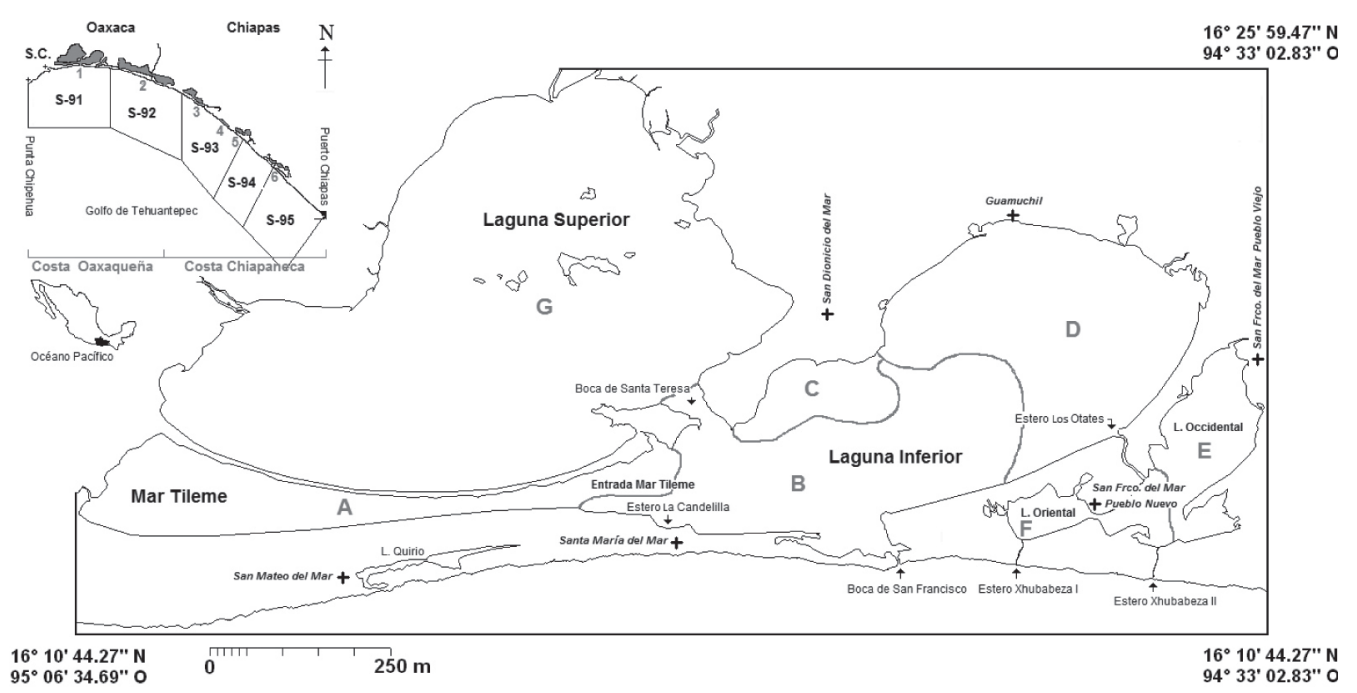

Fig. 1. Localización geográfica de la zona marina 90 de pesca en el GT, subzonas de pesca de la S-91 a la S-95. Los sistemas lagunares son: Huave (1), Mar Muerto (2), Cabeza de ToroLa Joya-Buenavista (3), Patos-Solo Dios (4), Carretas-Pereyra (5), Chantuto-Panzacola (6), Salina Cruz (S.C.). Localización geográfica de las zonas culturales de pesca en el Sistema Lagunar Huave: "Mareños"(A), "Fuerza del Pueblo"(B), "Playa Copalito"(C), "Guamuchil" (D), "Jaltepec de la Mar" (E) y "La Santa Rosa" (F), zona cultural de pesca Zapoteca (G). Tomado y modificado de Cervantes-Hernández et al. (2012)

Fig. 1. Geographic location of marine fishing zone 90 in the Gulf of Tehuantepec, subsectors S-91 to S-95. Coastal lagoon systems are: Huave (1), Mar Muerto (2), Cabeza de Toro-La Joya-Buenavista (3), Patos-Solo Dios (4), Carretas-Pereyra (5), Chantuto-Panzacola (6), Salina Cruz (S.C.). Geographic location of cultural fishing areas in the Huave Lagoon System: "Mareños" (A), "Fuerza del Pueblo" (B), "Playa Copalito" (C) "Guamuchil" (D), "Jaltepec de la Mar" (E) and "La Santa Rosa" (F). Zapoteca cultural fishing area (G). Taken and modified from Cervantes-Hernández et al. (2012) 
la presencia del Gobierno Federal y la intervención académica, por lo que hasta la fecha, no se ha podido consolidar, en coordinación con las sociedades cooperativas de producción pesquera Huave (SCPPH) y Zapoteca, un ordenamiento pesquero que permita mejorar la disponibilidad de los recursos pesqueros aún disponibles (camarón y peces) (Cervantes-Hernández et al. 2012). Por lo anterior, el SLH es uno de los sistemas lagunares menos estudiados a lo largo del Pacífico mexicano (Espinoza-Tenorio et al. 2011).

Entre el 2007 y 2008, el Gobierno Federal, con el apoyo de la Universidad del Mar, campus Puerto Ángel, Oaxaca, México, realizaron el Plan de Manejo Acuícola y Pesquero del SLH (Serrano-Guzmán, 2007), para recabar y analizar información histórica (en las zonas culturales de pesca y en cada SCPPH) (Fig. 1) acerca de la estructura de la flota de pesca y las tendencias de $\mathrm{C}_{\mathrm{t}}$ de los recursos camarón y peces. Adicionalmente, en el PMAPSLH se incluyó el primer análisis hidrológico para las zonas culturales de pesca Huave (excepto la Zapoteca) (Fig. 1). De entre las principales variables hidrológicas analizadas, se consideraron, e.g.: la salinidad (S en UPS), la temperatura superficial del agua (TSA en ${ }^{\circ} \mathrm{C}$ ) y la concentración de oxígeno disuelto (OD en $\mathrm{mg} / \mathrm{l}$ ).

Con la realización del PMAPSLH, se encontró que la flota de pesca estuvo constituida por cayucos de vela $(70 \%)$, cayucos con motor fuera de borda (de 5 a $25 \mathrm{HP})(20 \%)$ y lanchas con motor fuera de borda (entre 45 y 115 HP) (10\%); adicionalmente, es común a lo largo de la línea costera lagunar, la pesca a pie. Las artes de pesca utilizadas por los pescadores huaves registradas o no, incluyen: la red agallera, el chinchorro, la cimbra, atarrayas, líneas de mano y curricanes (Espinoza-Tenorio et al. 2011).

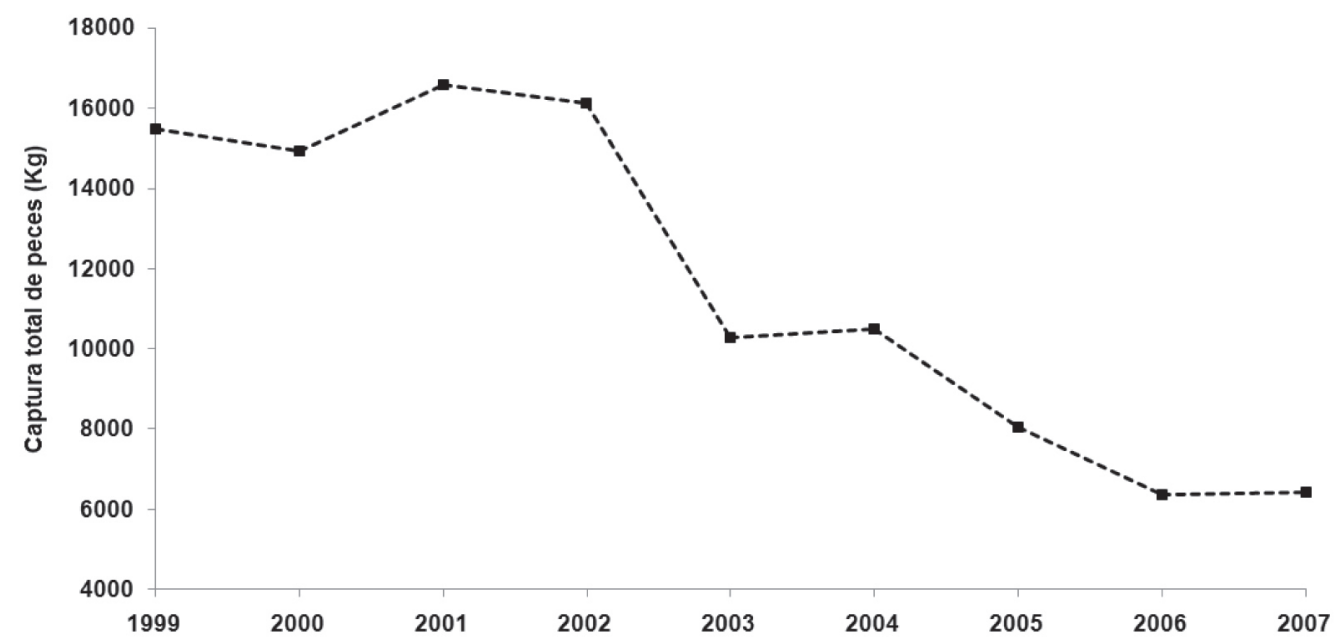

Fig. 2. Registros históricos para la captura lagunar del recurso peces en el Sistema Lagunar Huave de 1999 al 2007

Fig. 2. Historical records for lagoon catch of the fish resource in the Huave Lagoon System from 1999 to 2007 
Cervantes-Hernández et al. (2012) fueron los primeros en realizar el análisis integral de la información contenida en el PMAPSLH. Los autores estimaron un índice denominado "presión por pesca", para evaluar la disponibilidad potencial del recurso camarón en las zonas culturales de pesca Huave, así como el nivel de explotación sobre aquel, por parte de las SCPPH. Para la disponibilidad potencial, la "presión por pesca" incluyó los efectos combinados de la tasa de mortalidad natural (M) y el nivel óptimo de captura $\left(\mathrm{C}_{\mathrm{OPT}}\right)$. Para el nivel de explotación, se consideraron: el esfuerzo de pesca $\left(f_{\mathrm{i}}\right)$, el esfuerzo óptimo de pesca (fOPT) y la tasa de explotación (E).

En cada una de las zonas culturales de pesca Huave (Fig. 1), los parámetros antes mencionados se explicaron con la tendencia final que entre mayo y agosto de 2007, exhibió la variación hidrológica, así como el nivel de conservación de la cobertura de mangle (CervantesHernández et al. 2012). Los resultados fueron los siguientes: (a) las mejores condiciones hidrológicas (11.1 - 22.5 UPS, $27.0-28.6^{\circ} \mathrm{C}, 6.7-7.6 \mathrm{mg} / \mathrm{l}$ OD), con extensas áreas pobladas de mangle, se registraron en las lagunas Oriental y Occidental (Fig. 1), donde la disposición potencial del recurso camarón fue de $15000 \mathrm{~kg}$ entre 1999 y 2007 (en asociación con un alto nivel de explotación); (b) las peores condiciones hidrológicas (44.0 -54.7 UPS, 29.2 - $29.7^{\circ} \mathrm{C}, 5.0$ - $6.7 \mathrm{mg} / \mathrm{l} \mathrm{OD}$ ), con escasas áreas pobladas de mangle, se registraron en la Laguna Mar Tileme y áreas adyacentes (Fig. 1), donde la disposición potencial fue menor a 200 kg de 1999 al 2007 (en asociación con un bajo nivel de explotación).

El análisis integral realizado por Cervantes-Hernández et al. (2012) para el recurso camarón del SLH, aún no se ha hecho para el recurso peces, de manera que, bajo un mismo esquema hidrológico y de conservación del mangle, es necesario comprender las tendencias históricas de la disponibilidad potencial y el nivel de explotación, que el recurso peces exhibió en las zonas culturales de pesca Huave (Fig. 1). Por esta razón, se consideró utilizar al índice de "presión por pesca”, para generar comparaciones consistentes con respecto a las obtenidas para el recurso camarón; de manera que el trabajo aquí expuesto es la continuación del realizado por los autores antes citados.

\section{MATERIALES Y MÉTODOS}

En el Golfo de Tehuantepec (GT), al sur del Pacífico mexicano, se localiza la zona marina 90 de pesca (subdividida en cinco subzonas, de la S-91 a la S-95), delimitada entre Punta Chipehua, próxima a la ciudad de Salina Cruz, Oaxaca, México (16 01 ' 31.39" $\mathrm{N}$ y $95^{\circ} 22^{\prime} 24.56^{\prime \prime}$ O) y Puerto Chiapas, Chiapas, México (14 $40^{\circ}$ '55.81" $\mathrm{N}$ y $92^{\circ} 23$ '44.13" O) (CervantesHernández et al. 2010) (Fig. 1). Frente a la subzona S-91, se localiza el SLH (Fig. 1), que a través de la "Boca de San Francisco", tiene comunicación semipermanente con el GT (Fig. 1). El SLH está conformado de este a oeste por un conjunto de lagunas que son: Mar Ti- 
leme y áreas adyacentes, Laguna Quirio, Laguna Superior, Laguna Inferior, Laguna Oriental y Laguna Occidental. Las lagunas Superior e Inferior están comunicadas permanentemente por la "Boca de Santa Teresa". El estero Los Otates comunica permanentemente a la Laguna Inferior con las lagunas Oriental y Occidental, estas últimas poseen pequeños canales de comunicación permanentes con el GT y son, respectivamente, los esteros Xhubabeza I y II (CONGEASA, 1993) (Fig. 1).

Para el análisis poblacional, los datos utilizados fueron la $\mathrm{C}_{\mathrm{t}} \mathrm{de}$ peces $(\mathrm{kg})$ y el $\mathrm{f}_{\mathrm{i}}$ (días de pesca). Esta información fue proporcionada mediante registros mensuales de arribo de 1999 al 2007, por parte de las siguientes SCPPH: "Mareños" de San Mateo del Mar, "Fuerza del Pueblo" de Santa María del Mar, "Jaltepec de la Mar" de San Francisco del Mar Pueblo Viejo, "La Santa Rosa" de San Francisco del Mar Pueblo Nuevo, "Guamuchil" de Guamuchil y "Playa Copalito" de San Dionisio del Mar (Fig. 1). Todas aquellas contaron con el correspondiente registro de pesca expedido por la Sub-Secretaría de Pesca, Salina Cruz, Oaxaca, México.

Debido a la problemática sociocultural y a la implementación de zonas culturales de pesca (Fig. 1), en el SLH no existen especies objetivo de captura entre las SCPPH (EspinozaTenorio et al. 2011); por esta razón, y a pesar de que la captura de peces se registró con diferenciación entre especies comerciales (nombre común local), en los análisis subsiguientes, se consideró la integración de todas las especies de peces como único recurso. Lo antes mencionado se realizó al igual que en el caso del recurso camarón (Cervantes-Hernández et al. 2012), para el que no se pretendió realizar un análisis integral, sobre una especie de camarón en particular, sino que el interés fue a nivel de recurso.

Siguiendo el procedimiento descrito por Cervantes-Hernández et al. (2012), para cada SCPPH, se estimó la captura por unidad de esfuerzo (CPUE) (Ricker, 1975), que se representó con series de tiempo de 1999 al 2007. Cada serie de tiempo se desplegó y analizó visualmente, para localizar $n$ intervalos de tiempo en los cuales ocurrió un descenso mensual en la CPUE. Una vez localizados aquellos, se estimó el valor de la tasa de mortalidad total $(\mathrm{Z})$ con: $\ln (\mathrm{CPUE})=a-(\mathrm{Z} \cdot n)$ (Berry, 1967), donde $a$ es la ordenada al origen y $Z$ es la pendiente del modelo lineal. Por otra parte, para cada $n$ se cuantificó el esfuerzo de pesca promedio $\left(\mathrm{f}_{\text {iprom }}\right)$.

Con la información de los valores de la tasa $\mathrm{Z}$ y el $\mathrm{f}_{\text {iprom }}$ por serie de tiempo, se estimó para cada SCPPH, el valor de la tasa $\mathrm{M}$ con: $\mathrm{Z}=\mathrm{M}+(q$. $\mathrm{f}_{\text {iprom }}$ ) (Paloheimo, 1961), donde $q$ es la pendiente o coeficiente de capturabilidad (Ricker, 1975) (no utilizada en análisis subsiguientes) y $\mathrm{M}$ es la ordenada al origen del modelo lineal. La razón por la que el coeficiente $q$ no se utilizó en este trabajo es porque no se consideró a especies de peces objetivo. Por otra parte, la tasa de explotación se estimó con $\mathrm{E}=\mathrm{F} / \mathrm{Z}$, donde $\mathrm{F}$ es la tasa de mortalidad por pesca inscrita en $\mathrm{F}=\mathrm{M}$ - Z (Ricker, 1975). 
Con la variación mensual de la $\mathrm{C}_{\mathrm{t}}$ de peces y el $\mathrm{f}_{\mathrm{i}}$ entre 1999 y 2007 , se estimó por $\mathrm{SCPPH}$, la $\mathrm{C}_{\mathrm{OPT}}=$ $-0.25 \cdot\left(a^{2} / b\right)$ y el $\mathrm{f}_{\mathrm{OPT}}=-0.5 \cdot(a / b)$ (Schaefer, 1991), donde $a$ y $b$ son la ordenada al origen y la pendiente en el modelo lineal: $\mathrm{CPUE}=\mathrm{a}+(\mathrm{b}$. $\mathrm{f}_{\mathrm{i}}$ ). Adicionalmente, se estimaron los promedios mensuales para la $\mathrm{C}_{\mathrm{t}} \mathrm{de}$ peces y el $\mathrm{f}_{\mathrm{i}}$. La primera de aquellas se comparó con la magnitud de la $\mathrm{C}_{\mathrm{OPT}}$; mientras que la segunda se comparó con la magnitud resultante para la $\mathrm{f}_{\mathrm{OPT}}$. Para la estimación de la "presión por pesca" no se consideró a la $\mathrm{C}_{\mathrm{t}}$ de peces.

Al igual que Cervantes-Hernández et al. (2012), para estimar a la "presión por pesca", se utilizó un diseñó multivariado de correspondencia (Hair et al. 1999), que consta de los siguientes pasos:

1. Los valores esperados para M, $\mathrm{C}_{\text {OPT, }} \mathrm{f}_{\mathrm{i}}, \mathrm{f}_{\mathrm{OPT}} \mathrm{y} \mathrm{E}$ (denominados $i$ parámetros) se obtuvieron con: valor esperado de un $i$ parámetro = suma total de parámetros para una única $\mathrm{SCPPH} \cdot$ la frecuencia del $i$ parámetro en todas las SCPPH.

2. El residuo obtenido entre cada uno de los $i$ parámetros observados y esperados se estandarizó a valores de similitud con la distribución de $\mathrm{X}^{2}$ (Hair et al. 1999), grados de libertad $=(i-1) \cdot(j-1)$; donde, $i$ es el número de parámetros $\mathrm{y} j$ es el número de SCPPH.

3. Los valores de similitud se utilizaron para estimar a la "presión por pesca", que se asignó a cada $\mathrm{SCPPH}$, para visualizarlos en un gráfico perceptual de correspondencia (Hair et al. 1999). Para interpretar a la "presión por pesca", se consideró específicamente para el eje $\mathrm{X}$, el signo y la magnitud del valor de la similitud; de manera que aquella fue "baja" para valores de similitud negativos, "media" para valores de similitud altamente negativos cercanos a cero y "alta" para valores de similitud positivos mayores a cero.

\section{RESULTADOS}

Los valores esperados para los $i$ parámetros se muestran en el cuadro 1 .

En la zona cultural de pesca correspondiente a la SCPPH "Fuerza del Pueblo" (Fig. 1), se registró el valor más alto de la tasa $\mathrm{M}$, con el subsecuente descenso de la $\mathrm{C}_{\text {ort }}$ y la tasa E (Cuadro 1). En las zonas culturales de pesca correspondientes a las SCPPH "Guamuchil" y "Playa Copalito" (Fig. 1), se evidenciaron los valores más bajos de la tasa $\mathrm{M}$, con los subsecuentes incrementos de la $\mathrm{C}_{\text {orr }} \mathrm{y}$ la tasa $\mathrm{E}$ (Cuadro 1). Para todas las magnitudes antes mencionadas, estas evidenciaron valores intermedios en la zona cultural de pesca correspondiente a la SCPPH "La Santa Rosa" (Fig. 1, Cuadro 1).

Aproximadamente, las SCPPH invirtieron mensualmente el mismo $f_{i}$, sin embargo, el promedio mensual para la $\mathrm{C}_{\mathrm{t}}$ de peces extraída resultó diferente en cada una de aquellas; esto es: de 27 $\mathrm{kg}$ en "Mareños", de $83 \mathrm{~kg}$ en "Fuerza 
Cuadro 1. Valores esperados de los $i$ parámetros para las sociedades cooperativas de producción pesquera Huave (SCPPH) entre 1999 y 2007. Tasa de mortalidad natural (M, estimación mensual), nivel óptimo de captura $\left(\mathrm{C}_{\mathrm{OPT}}, \mathrm{kg}\right)$, promedio mensual del esfuerzo de pesca ( $f_{i}$, días de pesca), esfuerzo óptimo de pesca $\left(\mathrm{f}_{\mathrm{OPT}}\right.$, días de pesca), tasa de explotación (E, estimación mensual), promedio mensual de captura $\left(\mathrm{C}_{\mathrm{t}}, \mathrm{kg}\right)$, promedio anual de captura $\left(\mathrm{C}_{\mathrm{ta}}, \mathrm{kg}\right)$, parámetro no estimado (p.n.e.)

Table 1. Expected $i$ parameter values for the Huave fishing production cooperatives (SCPPH, acronym in Spanish) between 1999 and 2007. Natural mortality rate (M, monthly estimate), maximum sustainable yield $\left(\mathrm{C}_{\mathrm{OPT}}, \mathrm{kg}\right)$, monthly average fishing effort ( $\mathrm{f}_{\mathrm{i}}$, days of fishing), optimum fishing effort ( $\mathrm{f}_{\mathrm{OPT}}$, days of fishing), exploitation rate ( $\mathrm{E}$, monthly estimate), monthly average catch $\left(\mathrm{C}_{\mathrm{t}}, \mathrm{kg}\right)$, average annual catch $\left(\mathrm{C}_{\mathrm{ta}}, \mathrm{kg}\right)$, unestimated parameter (p.n.e., acronym in Spanish)

\begin{tabular}{lccccccc}
\hline \multicolumn{1}{c}{ SCPPH } & $\mathbf{M}$ & $\mathbf{C}_{\text {OPT }}$ & $\mathbf{f}_{\mathbf{i}}$ & $\mathbf{f}_{\text {OPT }}$ & $\mathbf{E}$ & $\mathbf{C}_{\mathbf{t}}$ & $\mathbf{C}_{\text {ta }}$ \\
\hline "Mareños" & p.n.e. & p.n.e. & 23 & p.n.e. & p.n.e. & 27 & 329 \\
"Fuerza del Pueblo" & 0.80 & 798 & 27 & 23 & 0.11 & 83 & 999 \\
"Jaltepec de la Mar" & p.n.e. & p.n.e. & 0 & p.n.e. & p.n.e. & 0 & 0 \\
"La Santa Rosa" & 0.19 & 4554 & 28 & 30 & 0.44 & 131 & 1583 \\
"Guamuchil" & 0.13 & 10777 & 30 & 37 & 0.46 & 774 & 9290 \\
"Playa Copalito" & 0.33 & 6069 & 30 & 30 & 0.45 & 396 & 4754 \\
\hline
\end{tabular}

del Pueblo", de $131 \mathrm{~kg}$ en "La Santa Rosa", de 774 kg en "Guamuchil" y de $396 \mathrm{~kg}$ en "Playa Copalito" (Cuadro 1). Las estimaciones mensuales del $f_{i}$ resultaron similares a su correspondiente homónimo de $\mathrm{f}_{\text {OPT. }}$ excepto para la SCPPH "Fuerza del Pueblo" (Cuadro 1).

En la zona cultural de pesca correspondiente a la SCPPH "Fuerza del Pueblo" (Fig. 1), la "presión por pesca" fue "baja" (similitud < - 0.5 ); en aquella, el nivel de explotación disminuyó, porque la disponibilidad potencial del recurso peces se redujo (Fig. 3). En las zonas culturales de pesca correspondientes a las SCPPH "Guamuchil" y "Playa Copalito" (Fig. 1), la "presión por pesca" fue "alta" (similitud entre 0 y 0.5 ); en aquellas, el nivel de explotación se incrementó, porque la disponibilidad potencial del recurso peces aumentó (Fig. 3). En la zona cultural de pesca correspondiente a la SCPPH "La Santa Rosa" (Fig. 1), la "presión por pesca" fue "media" (similitud entre -0.5 y 0 ), en aquella, el nivel de explotación y la disponibilidad potencial del recurso peces resultaron en magnitudes intermedias de similitud a las antes descritas (Fig. 3).

Los registros mensuales para la $C_{t}$ de peces y el $f_{i}$, que fueron proporcionados por las SCPPH "Mareños", resultaron insuficientes para estimar los $i$ parámetros inscritos en el cuadro 1. La SCPPH "Jaltepec de la Mar" no realizó la explotación de recurso peces en el SLH, ya que aquella no reportó ningún registro asociado a este rubro (Cuadro 1).

\section{DISCUSIÓN}

Para la estimación de los $i$ 


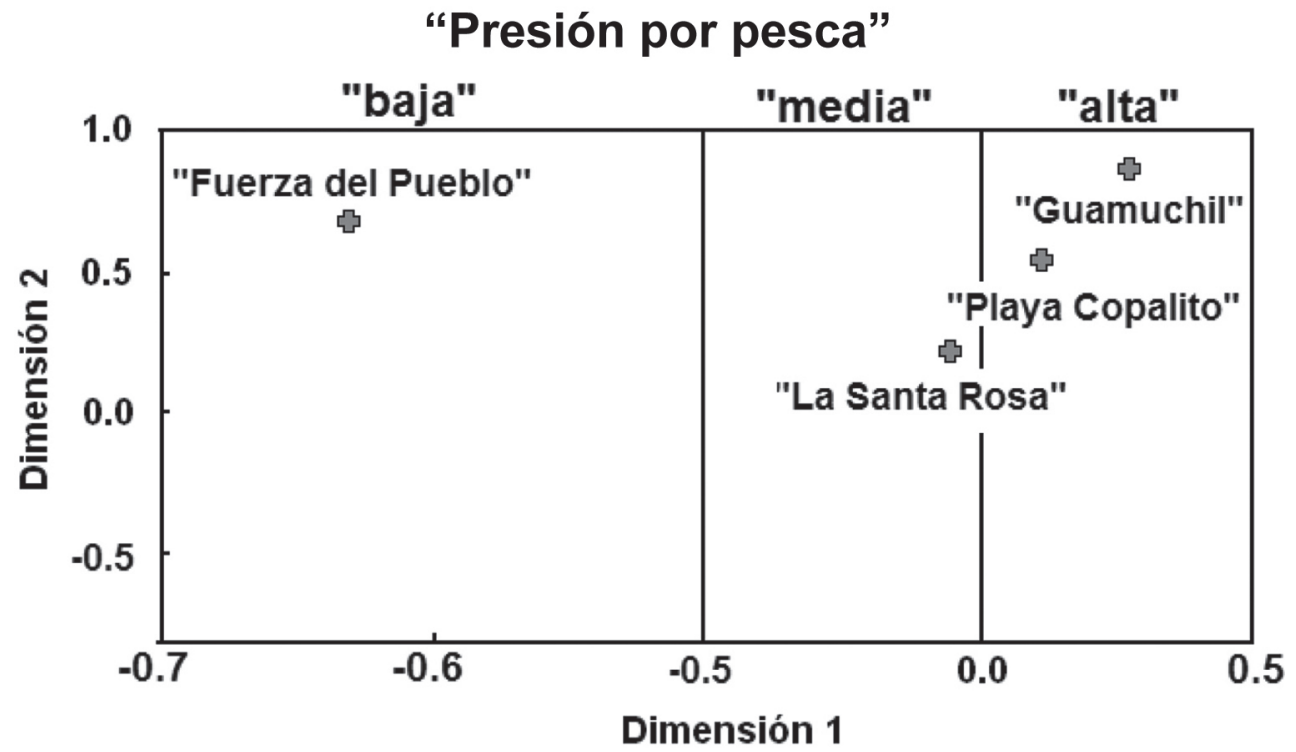

Fig. 3. Estimación de la "presión por pesca" para el recurso peces en cada una de las sociedades cooperativas de producción pesquera Huave de 1999 al 2007

Fig. 3. Estimated "fishing pressure" for the fish resource in each Huave fishing production cooperative from 1999 to 2007

parámetros inscritos en el cuadro 1 , se utilizó el $\mathrm{f}_{\mathrm{i}}$ en días de peca. En el SLH, un día de pesca varía dependiendo del tipo de embarcación utilizado (cayuco de vela, cayuco o lancha con motor fuera de borda), pero que persistentemente, aquella se realiza en un $70 \%$ con cayucos de vela (EspinozaTenorio et al. 2011). En el SLH, el día de pesca oscila entre 6 y $8 \mathrm{~h}$ de actividad pesquera y es la única unidad disponible inscrita en las hojas de arribo, por lo que no existe otra unidad registrada, con la que se pueda cuantificar al $\mathrm{f}_{\mathrm{i}}$. Debido a la falta de información con respecto a las características del poder de pesca para los tres tipos de embarcaciones que operan en el SLH, la "presión por pesca", al igual que en el caso del recurso camarón (Cervantes-Hernández et al. 2012), se estimó tomando como fuente principal de variación a la CPUE (Gulland, 1956; Shimada \& Schaefer, 1956; Beverton \& Holt, 1957; Allen \& Punsly, 1984; Hilborn \& Walters, 1992; Quinn \& Deriso, 1999; CervantesHernández et al. 2010).

Para estimar a la "presión por pesca", no se consideró el promedio mensual de la $\mathrm{C}_{t}$ de peces, porque la disponibilidad potencial para este recurso está más apegada al total que debió ocurrir según el valor de la $\mathrm{C}_{\mathrm{OPT}}$ (Hilborn \& Walters, 1992; Quinn \& Deriso, 1999; Cervantes-Hernández et al. 2006), en vez de lo reportado en las hojas de arribo, ya que como se mencionó anteriormente, en el SLH es común y persistente la pesca a pie $\left(\mathrm{C}_{t}\right.$ no registrada) (Espinoza-Tenorio et al. 2011); de manera que es inevitable la fuga de información pesquera. 
Para generar comparaciones consistentes con los resultados obtenidos por Cervantes-Hernández et al. (2012), se utilizaron los mismos modelos empleados por aquellos, que son los recomendados (Hilborn \& Walters, 1992; Punt \& Hilborn, 1996; Williams \& Prager, 2002; Chien-Hsiung, 2004) en el caso de análisis poblacionales sin énfasis a especies objetivo (modelos globales). Particularmente y debido a lo anterior, no se utilizaron modelos dinámicos de biomasa (modelos especialistas) (Cervantes-Hernández et al. 2010), porque no es posible asignar a todas las especies que conforman al recurso peces, un valor único de los parámetros dinámicos: tasa intrínseca de crecimiento poblacional (r), tamaño máximo de población $(\mathrm{k})$, capturabilidad (q) y biomasa inicial (Bo).

En la Laguna Mar Tileme y áreas adyacentes (Fig. 1), la hidrología reportada por Cervantes-Hernández et al. (2012) estuvo constituida por una alta $\mathrm{S}$ (de 44 a 54.7 UPS), TSA (de 29.2 a $30^{\circ} \mathrm{C}$ ) y bajos niveles de OD (de 5.0 a $6.7 \mathrm{mg} / \mathrm{l}$ ). Aquellas fueron persistentes a lo largo de la zona cultural de pesca correspondiente a la SCPPH "Mareños" (Fig. 1), extendiéndose hacia la perteneciente a la SCPPH "Fuerza del Pueblo" (Fig. 1). En esta última, el nivel de explotación para el recurso peces disminuyó $\left(\mathrm{f}_{\mathrm{OPT}}\right.$ $=23$ días de pesca, $\mathrm{E}=0.11$ mensual), porque la disponibilidad potencial de aquel se redujo $(\mathrm{M}=0.80$ mensual, $\mathrm{C}_{\text {OPT }}=798 \mathrm{~kg}$ ) (Cuadro 1), dando como resultado una "presión por pesca" "baja" para este recurso (Fig. 3). En las lagunas antes mencionadas y con una condición hidrológica tipificada como extrema,
Cervantes-Hernández et al. (2012) reportaron la menor disponibilidad potencial del recurso camarón a tasas $\mathrm{M}$ de entre 0.65 y 0.82 mensual, con una oscilación para la $\mathrm{C}_{\mathrm{OPT}}$ de $200 \mathrm{a} 477 \mathrm{~kg}$.

En las lagunas Oriental y Occidental (Fig. 1), la hidrología reportada por Cervantes-Hernández et al. (2012) resultó con gradientes opuestos a los reportados para la Laguna Mar Tileme y áreas adyacentes; estos fueron: 11.1 $22.5 \mathrm{UPS}, 27.0$ - $28.6^{\circ} \mathrm{C}, 6.7$ - $7.6 \mathrm{mg} / 1$ OD. Aquellas se registraron persistentes a lo largo de las zonas culturales de pesca correspondientes a las SCPPH "Jaltepec de la Mar" y "La Santa Rosa" (Fig. 1); y a través del estero Los Otates (Fig. 1), estas últimas se extendieron con gradientes descendentes (22.5 30.0 UPS, $27.0-28.1^{\circ} \mathrm{C},-7.6-8.0$ $\mathrm{mg} / \mathrm{l} \mathrm{OD})$, hacia las zonas culturales de pesca correspondientes a las SCPPH "Guamuchil" y "Playa Copalito" (Fig. 1). En aquellas últimas, el nivel de explotación se incrementó ( $\mathrm{f}_{\mathrm{OPT}}$ osciló de 30 a 37 días de pesca y E osciló entre 0.43 y 0.46 mensual), porque la disponibilidad potencial del recurso peces aumentó $\left(\mathrm{M}=0.13\right.$ mensual, $\mathrm{C}_{\mathrm{OPT}}$ osciló de 6069 a $10777 \mathrm{~kg}$ ) (Cuadro 1), dando como resultado una "presión por pesca" "alta" para este recurso (Fig. 3). Con respecto a la SCPPH "La Santa Rosa" (Fig. 1), el potencial de explotación y la disponibilidad potencial del recurso peces resultaron con magnitudes intermedias de similitud, generando una "presión por pesca" "media" (Fig. 3).

En las lagunas Oriental y Occidental (Fig. 1) y con una condición 
hidrológica tipificada como óptima (Cervantes-Hernández et al. 2012), se reportó la mayor disponibilidad potencial del recurso camarón a tasas $\mathrm{M}$ de entre 0.31 y 0.34 mensual, con una oscilación para la $\mathrm{C}_{\mathrm{OPT}}$ de 24247 a $29778 \mathrm{~kg}$. Tomando como referencia a las lagunas antes mencionadas, la disponibilidad potencial del recurso camarón descendió gradualmente, con dirección hacia la Laguna Mar Tileme y áreas adyacentes.

Con base en lo antes descrito, se concluye que el recurso peces evidenció un gradiente descendente, según su disponibilidad potencial, que va desde las zonas culturales de pesca ubicadas en la Laguna Inferior, hasta las localizadas en la Laguna Mar Tileme y áreas adyacentes (Fig. 1). En aquella última, donde la hidrología fue persistentemente extrema (CervantesHernández et al. 2012), la "presión por pesca" sobre el recurso peces resultó "baja" (Fig. 3); mientras que en la primera, donde la hidrología fue persistentemente "media" (CervantesHernández et al. 2012), la "presión por pesca" sobre el recurso peces resultó "alta" (Fig. 3). Donde la hidrología fue persistentemente óptima (CervantesHernández et al. 2012), la "presión por pesca" sobre el recurso peces resultó "media" (Fig. 3).

La SCPPH "Playa Copalito" se encontró enteramente dedicada a

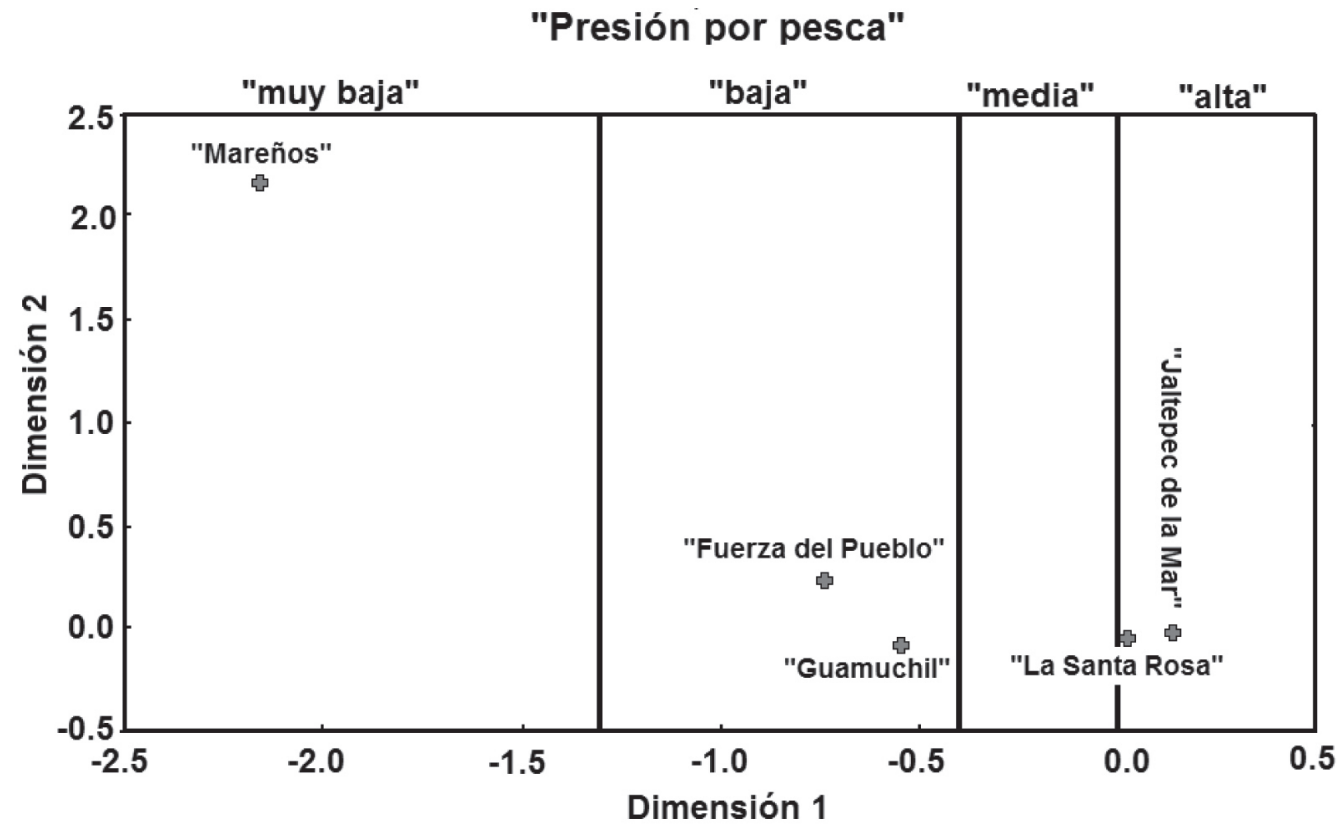

Fig. 4. Estimación de la "presión por pesca" para el recurso camarón en cada una de las sociedades cooperativas de producción pesquera Huave de 1999 al 2007 (CervantesHernández et al. 2012)

Fig. 4. Estimated "fishing pressure" for the shrimp resource in each Huave fishing production cooperative from 1999 to 2007 (Cervantes-Hernández et al. 2012) 
la pesca del recurso peces (Fig. 3); mientras que la SCPPH "Guamuchil" se evidenció con una "presión por pesca" "alta" sobre el recurso peces (Fig. 3) y "baja" sobre el recurso camarón (Fig. 4) (Cervantes-Hernández et al. 2012). La SCPPH "La Santa Rosa" se evidenció con una "presión por pesca" "media" sobre el recurso peces (Fig. 3) y "alta" sobre el recurso camarón (Fig. 4) (Cervantes-Hernández et al. 2012). La SCPPH "Jaltepec de la Mar" se reportó enteramente dedicada a la pesca del recurso camarón (Fig. 4) (CervantesHernández et al. 2012). En concordancia con los autores antes citados, en la Laguna Mar Tileme y áreas adyacentes, persistentemente se evidenció una baja disposición potencial de los recursos camarón ("presión por pesca" "muy baja") (Fig. 4) y peces ("presión por pesca" "baja") (Fig. 3).

Mediante la aplicación de encuestas en la comunidad Huave de San Mateo del Mar (Fig. 1), RoblesZavala (2004) generó un modelo socioeconómico constituido por cinco índices, con cada uno de aquellos, se evaluó un tipo de capital, e.g.: humano, físico, natural, social y financiero. Los diferentes tipos de capitales se escalaron entre cero (poco satisfactorio) y 10 (muy satisfactorio) (Fig. 5). Por otra parte, con la combinación multivarida de aquellos, el autor evaluó el nivel de bienestar integral, en este caso, representado por la amplitud en la apertura de diamantes socioeconómicos (Fig. 5). Para el caso que compete a este trabajo, el capital natural es particularmente importante, ya que resultó ser un indicador del estado de conservación del entorno natural y que para el caso de la comunidad Huave de San Mateo del Mar, aquel en 1999, resultó con una escala 3 (Fig. 5a).

Con los mismos procedimientos socioeconómicos antes descritos, Robles-Zavala (2014) reportó en el 2007, una escala 6 para el capital natural en la comunidad Huave de San Dionisio del Mar (Fig. 5b); mientras que, para el municipio conformado por las comunidades Huave San Francisco del Mar Pueblo Viejo y Pueblo Nuevo, aquel resultó con una escala 9 (Fig. 5c).

Anterior al PMAPSLH, los trabajos socioeconómicos antes descritos, advirtieron acerca de una diferenciación geográfica con respecto a la conservación

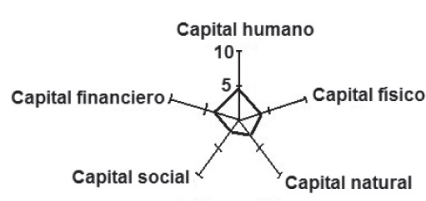

(a)

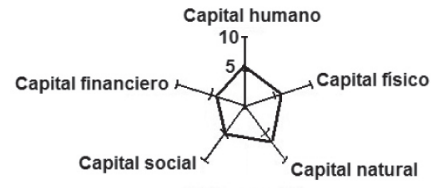

(b)

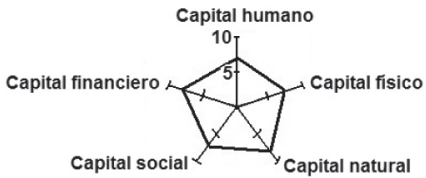

(c)

Fig. 5. Modelo socioeconómico para evaluar el bienestar integral en las comunidades Huave: San Mateo del Mar (a), San Dionisio del Mar (b) y San Francisco del Mar Pueblo Viejo y Pueblo Nuevo (c) (Robles-Zavala, 2004; 2014)

Fig. 5. Socio-economic model used to assess the integral well-being of the Hueve communities: San Mateo del Mar (a), San Dionisio del Mar (b) and San Francisco del Mar Pueblo Viejo and Pueblo Nuevo (c) (Robles-Zavala, 2004; 2014) 
del entorno natural en el SLH (excepto la zona Zapoteca), que resultó consistente y vigente en relación con el análisis hidrológico reportado por CervantesHernández et al. (2012).

En la comunidad Huave de San Mateo del Mar, el bienestar integral resultó bajo (Robles-Zavala, 2004) (Fig. 5a), coincidiendo con una baja disposición potencial para los recursos camarón y peces (Figs. 3 y 4). En la comunidad Huave de San Dionisio del Mar, el bienestar integral resultó medio (Robles-Zavala, 2014) (Fig. 5b), coincidiendo con una baja disposición potencial para el recurso camarón y alta para el recurso peces (Figs. 3 y 4 ). Para el municipio conformado por las comunidades Huave San Francisco del Mar Pueblo Viejo y Pueblo Nuevo, el bienestar integral resultó alto (RoblesZavala, 2014) (Fig. 5c), coincidiendo con una alta disposición potencial de los recursos camarón y media para el recurso peces (Figs. 3 y 4).

Es necesario mencionar que los episodios de apertura y cierre de la "Boca de San Francisco" no han sido documentados en su totalidad. Los pescadores de las SCPPH indicaron que aquella estuvo cerrada de mayo de 2006 a septiembre de 2010. Durante este período denominado "Boca Cerrada", Cervantes-Hernández et al. (2012) reportaron la hidrología de mayo a agosto-octubre de 2007; mientras que la estimación de la "presión por pesca" para el recurso peces cubrió 7.4 años del período denominado "Boca Abierta" (de 1999 a abril de 2005) y 1.8 años en "Boca Cerrada" (de mayo de 2005 al
2007). Para poder asociar a la "presión por pesca" con la hidrología reportada por Cervantes-Hernández et al. (2012), aquella se utilizó como un producto de tendencia final, con el predominio del período "Boca Cerrada". Pero de acuerdo con Robles-Zavala (2004; 2014) (Fig. 5), se observó indirectamente, que la hidrología con el predominio del período "Boca Abierta", fue aproximadamente la misma a la reportada por CervantesHernández et al. (2012).

La "presión por pesca" estimada para el recurso peces puede ser utilizada confiablemente para la realización de trabajos futuros, encaminados a comprender el nuevo rumbo que está tomando la pesquería marinolagunar en el SLH. Adicionalmente, se recomienda monitorear el recurso peces, integrando la captura que resulte de la zona Zapoteca. Como dato adicional, la "Boca de San Francisco" ha permanecido abierta desde septiembre de 2010 hasta la fecha.

\section{AGRADECIMIENTOS}

Este trabajo fue desarrollado en la Universidad del Mar, Puerto Ángel, Oaxaca, con el apoyo económico del PMAPSLH.Agradecemosampliamente los comentarios y la revisión hecha por los revisores anónimos.

\section{BIBLIOGRAFÍA}

Allen, R. \& Punsly, R. (1984). Catch rates as indices of abundance of yellowfin tuna, Thunnus albacares, in the eastern Pacific Ocean. Bull. I-ATTC., 18, 301-379.

Berry, R. (1967). Dynamic of the Tortugas (Florida) pink shrimp population. 
Disertación de doctorado no publicada, University of Rhode Island, EE.UU.

Beverton, H. \& Holt, S. (1957). On the dynamics of exploited fish population. Nueva York, EE.UU.: Chapman \& Hall. Bozada-Robles, R. L. (2002). Los pescadores zapotecas del complejo lagunar del istmo de Tehuantepec, Oaxaca, México. Recuperado en febrero 12, 2011, disponible en http://www.ciesasgolfo.edu.mx/istmo/docs/pescadoreszapotecas/indice.htm

Cervantes-Hernández, P., Ramos-Cruz, S. \& Gracia, A. (2006). Evaluación del estado de la pesquería de camarón en el Golfo de Tehuantepec. Hidrobiol., 16(3), 233-239.

Cervantes-Hernández, P., Flores-Gómez, A., Serrano-Guzmán, S. J., RamosCruz, S. \& Gallardo-Berumen, M. I. (2010). Historical exploitation and evaluation of brown shrimp fishery Farfantepeneaeus californiensis (Decapoda, Dendrobranchiata) in the Gulf of Tehuantepec, Oaxaca, Mexico. $\mathrm{Pa}$ namjas., 5(4), 486-494.

Cervantes-Hernández, P., Gallardo-Berumen, I. \& Serrano-Guzmán, S. (2012). Explotación y distribución de la captura artesanal de camarón en el sistema lagunar Huave, Oaxaca, México. Rev. Mar. Cost., 4, 67-81.

Chien-Hsiung, W. (2004, August). Improvement of the Schaefer model and its application. Conference. 17th Meeting of the Standing Committee on Tuna and Billfish. Majuro, Marshall Islands. CONGEASA. (1993). Caracterización biológica pesquera del camarón en la Laguna Oriental, Occidental, Inferior y marismas del Estado de Oaxaca. Oaxaca, México: Sociedad Cooperativa de Producción Pesquera Jaltepec de la Mar, Salina Cruz, Oaxaca, México.
Espinoza-Tenorio,A., Espejel, I., Matthias, W. \& Zepeda-Domíguez, J. A. (2010). Contextual factors influencing sustainable fisheries in Mexico. Mar. Policy, 35, 343-350. doi: http://dx.doi. org/10.1016/j.marpol.2010.10.014

Espinoza-Tenorio, A., Bravo-Peña, L. C., Serrano-Guzmán, J. S., Ronsón-Paulín, J. A., Ahumada, M. A., Cervantes-Hernández, P., Robles-Zavala, E., Fuentes, M. P. \& Gallardo-Berumen, M. I. (2011). La diversidad étnica como factor de planeación pesquera artesanal: Chontales, Huaves y Zapotecas del Istmo de Tehuantepec, Oaxaca, México. En G. Alcalá \& A. Camargo (Eds.), Pescadores en América Latina y el Caribe: espacio, población, producción y política, vol. I (pp. 167216). México, D. F.: Facultad de Ciencias, Unidad Multidisciplinaria de Docencia e Investigación - SISAL, Universidad Nacional Autónoma de México.

Espinoza-Tenorio, A., Wolff, M., Espejel, I. \& Montaño-Moctezuma, G. (2013). Using Traditional Ecological Knowledge to Improve Holistic Fisheries Management: Transdisciplinary Modeling of a Lagoon Ecosystem of Southern Mexico. Ecol. Soc., 18(2), 1-18.

Gulland, J. A. (1956). On the fishing effort in English demersal trawl fisheries. Fish. Invest., I., 20(5), 1-41.

Hair, J. F., Anderson, R. E., Tatham, R. L. \& Black, W. C. (1999). Multivariate data analysis. Iberia, Madrid: Prentice Hall.

Hilborn, R. \& Walters, C. J. (1992). Quantitative Fisheries Stock Assessment: Choice, Dynamics and Uncertainty. Londres, Inglaterra: Chapman \& Hall. doi: http://dx.doi. org/10.1007/978-1-4615-3598-0

Millán, S. (2003). Huaves. En C. EnriqueSerrano \& L. Cruz-González (Eds.), 
Pueblos indígenas del México contemporáneo. México, D. F.: CDI/PNUD.

Paloheimo, J. (1961). Studies on estimation of mortalities, comparison of a method described by Beverton and Holt and a new linear formula. J. Fish. Res. Bd. Can., 18(5), 645-662. doi: http://dx.doi.org/10.1139/f61-050

Punt, A. E. \& Hilborn, R. (1996). Biomass dynamic models: User's Manual. Computarized Information Series (Fisheries) No. 10. Roma, Italia: FAO.

Quinn, T. J. \& Deriso, R. B. (1999). Quatitative Fish Dynamics. New York, EE.UU.: Oxford University Press.

Ricker, W. E. (1975). Computation and interpretation of biological statistics of fish populations. Bull. Fish. Res. Board Can., 191, 1-382.

Robles-Zavala, E. (2004). Poverty in Rural Fishing Communities: A View from the Inside. Disertación de doctorado. School for International Development, University of East Anglia, Inglaterra.

Robles-Zavala, E. (2014). Estudio de pobreza y bienestar en la costa de Oaxaca a través del Enfoque de Medios de Vida Sustentables. En S. V. Foucat-Avila (Ed.), Medios de vida sustentables y capitales en comunidades rurales. México, D. F.: Editorial Porrúa.

Schaefer, M. B. (1991). Some aspects of the dynamics of populations important to the management of the commercial marine fisheries. Bull. Mat. Biol., 53(1/2), 253-279. doi: http://dx.doi. org/10.1007/BF02464432

Serrano-Guzmán, S. J. (2007). Plan de Manejo Acuícola y Pesquero del Sistema Lagunar Huave. Reporte técnico. Oaxaca, México: Comisión Nacional de Acuacultura y Pesca, Sub-Secretaría de Pesca Salina Cruz, Oaxaca, México, Universidad del Mar, campus Puerto Ángel.

Shimada, B. M. \& Schaefer, M. B. (1956). A study of changes in fishing effort, abundance, and yield for yellowfin and skipjack tuna in the eastern tropical $\mathrm{Pa}$ cific Ocean. Bull. I-ATTC., 1, 351-421.

Williams, E. H. \& Prager, M. H. (2002). Comparison of equilibrium and nonequilibrium estimators for the generalized production model. Can. J. Fish. Aquat. Sci., 59(9), 1533-1552. doi: http://dx.doi.org/10.1139/f02-123 